\title{
Manipulation of optically fabricated particle arrays using broadband radiation
}

\author{
Justo Rodríguez and David L. Andrews \\ Nanostructures and Photomolecular Systems, School of Chemical Sciences, \\ University of East Anglia, Norwich NR4 7TJ, United Kingdom
}

\begin{abstract}
Consideration is given to methods of manipulating optically fabricated particle arrays using broadband radiation and a superposition of optical fields. Specifically, the changes that the optical binding energy experiences, when part of the spectrum of this light is filtered, are analyzed. It is then shown that these optically induced arrays can be reordered by the introduction of additional fields with transverse Poynting vectors. Subsequently, it is shown how pairs of particles can be reordered on a surface by modifying the form of the optical binding interaction. Finally, the effect of particle size on these methods is briefly discussed.
\end{abstract}

Keywords: Optical binding, optical manipulation, potential energy landscapes, Rayleigh particles, continuum radiation

\section{INTRODUCTION}

In the presence of intense optical fields, inter-particle interactions are induced that are fundamentally similar to the Casimir-Polder potential $[1,2]$. This phenomenon, now usually called optical binding, was originally predicted from calculations based on quantum electrodynamics (QED) [3] and almost a decade later it was first observed experimentally [4]. It is currently a subject of significant experimental and theoretical interest [1, 2, 5-29], particularly in connection with the capacity to produce particle structures. The arrays of Rayleigh particles that can be formed in plane-waves have been subject of several recent studies [1, 2, 21-23]. It has also been shown that particle arrays in such optical traps may be molded by irradiating with secondary optical fields [19].

Optical binding is clearly observed in super-continuum laser fields, circumventing the problems that can otherwise arise with coherent monochromatic radiation, due to interference in the optical trap [20,28]. The continuum nature of light has also been theoretically shown to find applications in the fabrication of mouldable particle arrays [1]. Here we extend these studies and explore a variety of means for modifying the optical binding potential energy landscapes, by superimposing these highly incoherent optical fields. Specifically, we begin by extending previous optical binding studies to exhibit the effect of filtering part of the broadband radiation, on the energy surface responsible for the formation of two-dimensional arrays. Subsequently, it is shown how the energy surface is modified by means of secondary laser fields with transverse Poynting vectors, and the dependence in form of such a surface on the polarization of the secondary field is explored. Techniques for the manipulation of particles laying on flat surfaces are also investigated. Finally, the effect of particle size on these manipulation techniques is briefly discussed in the conclusions.

\section{OPTICAL BINDING IN BROADBAND SPECTRUM LASER FIELDS}

By reducing or increasing the filtered region of a broadband spectrum light using long-wavelength pass filters (i.e. by changing $\omega_{1}$ as shown in Fig. 1) optically induced particle chains formed along the Poynting vector of an optical field may be expanded and contracted. Here we consider the effect of this spectrum alteration on the optical binding energy surface determining two-dimensional arrays of spherical particles. We begin by writing the binding energy, derived using the electric dipole approximation, in a continuum state of light $|\{x\}\rangle[1]$ :

Optical Trapping and Optical Micromanipulation VI, edited by Kishan Dholakia, Gabriel C. Spalding, Proc. of SPIE Vol. 7400, 74001I - (c) 2009 SPIE · CCC code: 0277-786X/09/\$18 - doi: 10.1117/12.825283 


$$
\left\langle\Delta E_{\text {avg }}\right\rangle=\operatorname{Re}\left[\frac{\varepsilon}{2 c} \int S(\omega) V_{z z}(\omega, \mathbf{R})\left(\chi^{(A)}(\omega) \chi^{(B)}(-\omega) e^{i\left(\frac{\omega\left(r_{y}^{(A)}-r_{y}^{(B)}\right)}{c}\right)}+\chi^{(A)}(-\omega) \chi^{(B)}(\omega) e^{-i\left(\frac{\omega\left(r_{y}^{(A)}-r_{y}^{(B)}\right)}{c}\right)}\right)\right] d \omega
$$

where the interacting particles are Rayleigh spheres with electric susceptibility $\chi^{(\xi)}(k)=\chi^{(\xi)}(k,-k)$. The optical field is assumed to be a plane-wave propagating along the $y$-axis and with polarization along the $z$-axis. The elements of the coupling tensor are;

$$
V_{i j}(\omega, \mathbf{R})=n\left(\frac{n^{2}+2}{3}\right)^{2} \frac{e^{i \frac{n \omega}{c} R}}{4 \pi \varepsilon n^{3} R^{3}}\left[\left(1-i \frac{n \omega}{c} R\right)\left(\delta_{i j}-3 \frac{R_{i} R_{j}}{R^{2}}\right)-\left(\frac{n \omega}{c} R\right)^{2}\left(\delta_{i j}-\frac{R_{i} R_{j}}{R^{2}}\right)\right],
$$

where $n$ is the refractive index of the medium and, again in (1);

$$
S(\omega)=\left[\frac{c \hbar \omega\left\langle\{x\}\left|a^{\dagger}(\omega) a(\omega)\right|\{x\}\right\rangle}{V}\right]
$$

$a(\omega)$, and $a^{\dagger}(\omega)$ being the photon continuum annihilation and creation operators, respectively [31]; $V$ is an arbitrary quantization volume.

We now determine the energy surface, with some assumptions on the state of the system. First, for convenience the spectral irradiance of the light is assumed to have Lorentzian form:

$$
S(\omega)=\frac{K}{\left(\omega_{0}-\omega\right)^{2}+\gamma^{2}},
$$

where $\omega_{0}$ determines the spectral position of maximum irradiance; the parameter $K$ determines the intensity of the light and $\gamma$ the bandwidth. For simplicity, each of the interacting particles, $A$ and $B$, is assumed to comprise centres whose essentially two-level optical response is dominated by the ground state, $|0\rangle$, and one excited state, $|r\rangle$. Consequently the particle susceptibilities are given by:

$$
\chi^{(A)}(\omega)=\chi^{(B)}(\omega)=\frac{2 N}{3 \hbar} \frac{\omega_{r 0}^{2}\left|\mu^{r 0}\right|^{2}}{\varepsilon\left(\omega_{r 0}{ }^{2}-\omega^{2}\right)},
$$

where $N$ is the number of optical centers per particle; $\hbar \omega_{r 0}$ is the difference between the energy of the states $|0\rangle$ and $|r\rangle$; $\mu^{r 0}=\langle r|\mu| 0\rangle$ is the transition electric dipole moment associated with the electronic transition $|r\rangle \rightarrow|0\rangle$. To preclude absorption and any attendant thermal effects (which might change optical properties), and in order to observe the dependence of the inter-particle potential energy surfaces on spectrally different forms of irradiance, part of the light is filtered. Experimentally, this is expedited by the use of long-pass filters, whose effect on the spectral irradiance is illustrated in Fig. 1. Under these conditions, and assuming the particles are optically trapped in vacuum, the interparticle potential energy for each particle pair can be written as follows: 


$$
\left\langle\Delta E_{\text {avg }}\right\rangle=\operatorname{Re}\left[\frac{4 K N^{2}\left|\mu^{r 0}\right|^{4} \omega_{r 0}^{2}}{9 c \hbar^{2} \varepsilon_{0}} \int_{0}^{\omega_{1}} \frac{1}{\left(\omega_{r 0}{ }^{2}-\omega^{2}\right)^{2}} \frac{V_{z z}(\omega / c, \mathbf{R})}{\left(\omega_{0}-\omega\right)^{2}+\gamma^{2}} \cos \left(\frac{\omega\left(r_{y}^{(A)}-r_{y}^{(B)}\right)}{c}\right) d \omega\right] .
$$

The integral can be evaluated numerically. It is now possible to explore the effect of choosing different spectral intervals by using pass filters with different wavelength characteristics (hence different values of $\omega_{1}$ ).

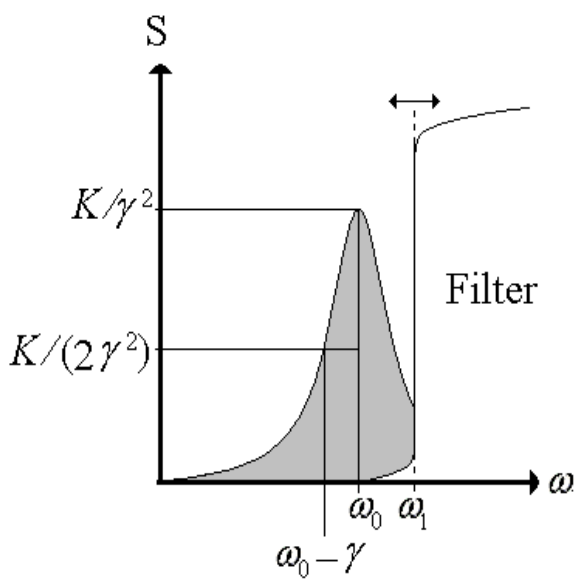

Figure 1. Spectral irradiance of light, with Lorentzian spectrum, transmitted through a long-wavelength pass filter, see equation (4).
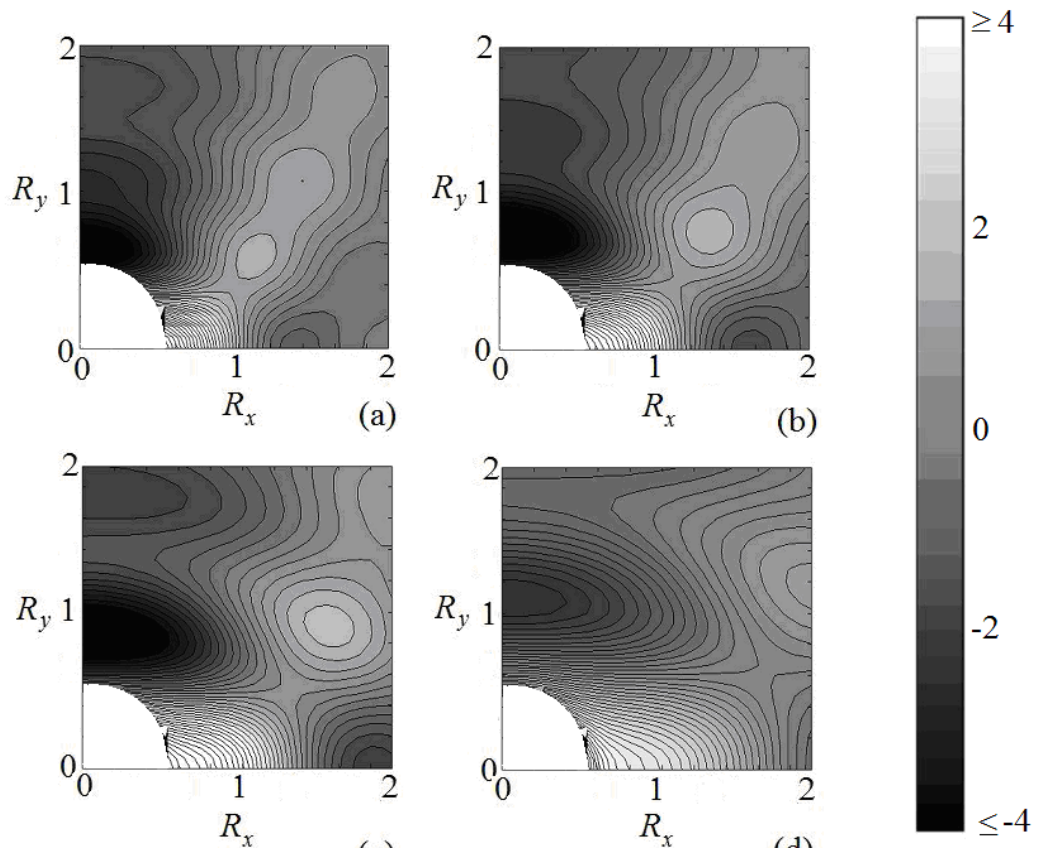

(c)
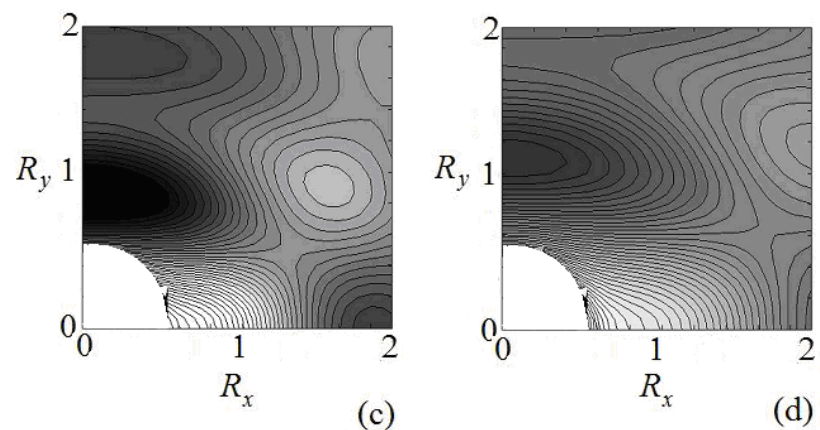

Figure 2. Optical binding energy surface, along the Poynting vector of the beam ( $y$ axis), given by equation (11) for $\omega_{0}=3 \gamma$, $\omega_{r 0}=10 \gamma$, and (a) $\omega_{1}=6 \gamma$ (b) $\omega_{1}=5 \gamma$ (c) $\omega_{1}=4 \gamma$ (d) $\omega_{1}=3 \gamma$. The units of energy and distance are $100 K N^{2}\left|\mu^{r 0}\right|^{4} /\left(c \varepsilon_{o} \hbar^{2}\right)$ and $c /(\gamma)$, respectively. 
The graphs in Fig. 2 show the optical binding energy surface on an $x y$-plane, transverse to the polarization of the field, for different values of $\omega_{1}$. As has been proven in a previous investigation [22], particles are trapped on this plane and organized within it by optical binding forces. These contour maps reveal that by reducing the region of the spectrum interacting with the particles (i.e. by reducing $\omega_{1}$ ), the minima on the energy surface becomes more similar to the one induced by a single frequency light [22]. This is as might be anticipated, due to the fact that the spectrum becomes less broad. Also with this change of $\omega_{1}$, the energy minima along the $y$-axis and along the $x$-axis are displaced, increasing the achievable length of stable particle arrays transverse and longitudinal to the Poynting vector. The graphs reveal that any array of Rayleigh particles formed by optical binding may be controllably extended or contracted by filtering appropriate frequency regions of the continuum input radiation.

\section{OPTICAL BINDING WITH A SECONDARY BROADBAND FIELD}

A recurrent motif in studies of optical binding is the use of counter-propagating laser beams. This is a device to ensure that beam radiation pressure cancels out, such that the formation of particle arrays is determined by their individual and collective interactions with the electromagnetic fields [7]. Here, we consider one such laser set-up, supplemented by the addition of a secondary laser throughput designed to modify the total field the particles experience. Fig. 3 illustrates the wave-vector and polarization vector geometry, also defining parameters for establishing orientations relative to the interparticle displacement vector. A similar set-up has been the subject of recent studies [19], but it is now shown that the orientation of polarization in the secondary field plays a crucial role in the morphology of the created particle arrays. Two counter-propagating beams (primary beams $\mathbf{k}_{1}$ and $-\mathbf{k}_{1}$, the standard configuration) are located on the $y$-axis, each having the same polarization in the $z$ direction. These beams are intersected orthogonally by secondary fields, $\mathbf{k}_{2}$ and - $\mathbf{k}_{2}$, propagating along the $x$-axis. Two cases are independently considered in this section: in one of them, the polarization vector $\mathbf{E}_{2}$ is on the $z$-axis, and in the second case it is disposed along the $x$-axis. The net field the particles experience, in the region where the lasers cross, can then be adapted by controlling the relative irradiance of the primary and secondary beams. As will be shown, the resulting optical perturbation of the particles determines the way they are arranged.

When, as in Fig. 3, four plane-waves are taken into account, the following expression emerges for the optical binding energy in vacuum;

$$
\begin{aligned}
\Delta E= & \left(\frac{1}{4 \pi c^{4}}\right) \int_{0}^{\omega_{1}} \chi^{(A)} \chi^{(B)}\left(\left[S_{s}(\omega) D_{1}+S_{p}(\omega) C_{1}\right] \frac{c \cos \frac{n \omega}{c} R}{n \omega R}+\right. \\
& {\left.\left[S_{s}(\omega) D_{3}+S_{p}(\omega) C_{3}\right]\left(\frac{c^{3} \cos \frac{n \omega}{c} R}{(n \omega R)^{3}}+\frac{c^{2} \sin \frac{n \omega}{c} R}{(n \omega R)^{2}}\right)\right) \omega^{3} d \omega . }
\end{aligned}
$$

Here $S_{p}(\omega)$ and $S_{s}(\omega)$ are the spectral irradiances of the primary and secondary counter-propagating beam pairs, respectively; these spectra are assumed to be of Lorentz form with identical width, $\gamma$, and position of the maxima, $\omega_{0}$, but with different intensities quantified by the parameters $K_{p}$, for the primary beam pair, and $K_{s}$ for the secondary pair; also $C_{n}=\left(1-n\left(R_{z} / R\right)^{2}\right) \cos \left(k R_{y}\right)$ and $D_{n}=(1-n M) \cos \left(k R_{x}\right)$, where $M=\left(R_{x} / R\right)^{2}$ when $\mathbf{E}_{2}$ is parallel to the $y$-axis and $M=\left(R_{z} / R\right)^{2}$ when $\mathbf{E}_{2}$ is parallel to the $z$-axis; the susceptibilities are real and again given by equation (5). Finally, $R_{x}, R_{y}$ and $R_{z}$ are the elements of the vector position $\mathbf{R}$ of one particle respect to the other, and the magnitude of the vector is $R=\left[\left(R_{x}\right)^{2}+\left(R_{y}\right)^{2}+\left(R_{z}\right)^{2}\right]^{1 / 2}$. 


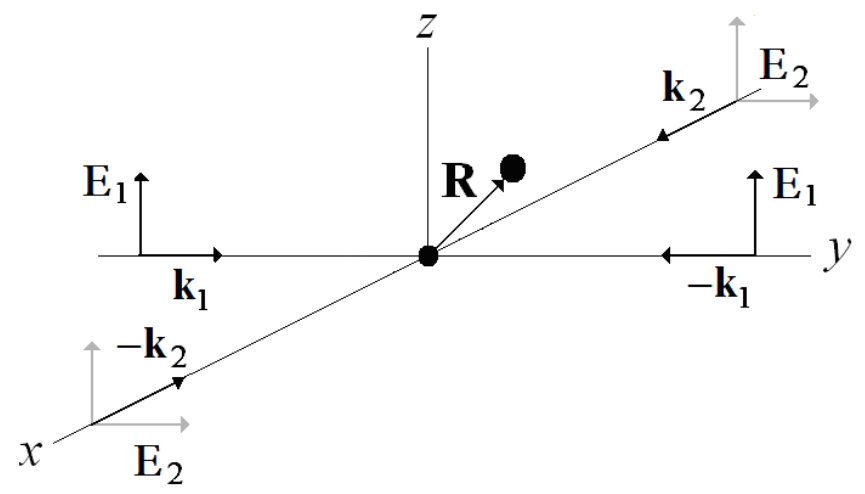

Figure 3. Laser set-up. Particles are trapped in a primary pair of counter-propagating optical fields, $\left(\mathbf{E}_{1}, \mathbf{k}_{1}\right)$ and $\left(\mathbf{E}_{1},-\mathbf{k}_{1}\right)$, and the particle array is modified by increasing the irradiance of the secondary pair of fields, $\left(\mathbf{E}_{2}, \mathbf{k}_{2}\right)$ and $\left(\mathbf{E}_{2},-\mathbf{k}_{2}\right)$. The polarization vectors in gray represent two cases considered; in one case, $\mathbf{E}_{2}$ is parallel to the $z$-axis and in the second it is parallel to the $y$-axis.

The effect of increasing the magnitude of the irradiance of the secondary beams, with $\mathbf{E}_{2}$ along the $y$-axis, is exhibited in Fig. 4, where the contour maps (a), (b), (c), and (d) show the surface of the optical binding energy on the $x y$-axis for $K_{s} / K_{p}=0.2, K_{s} / K_{p}=0.4, K_{s} / K_{p}=0.6$, and $K_{s} / K_{p}=0.8$, respectively. In Fig. 4 (a), the irradiance of the primary beam pair dominates, and the potential surface is similar to the one observed when there is no secondary field, as in Fig. 2 (a). Through a progressive increase in irradiance of the secondary field, Fig. 4 (b), (c), and (d), the first potential well along the $y$-axis becomes deeper and the energy minimum moves to the near-field zone. Consequently if a particle pair is initially bound by the first energy minimum along the $y$-axis and the irradiance of the secondary field is increased, the particle pair is attracted to the near-field zone. Along the $x$-axis, no significant change is observed in the potential energy surface.
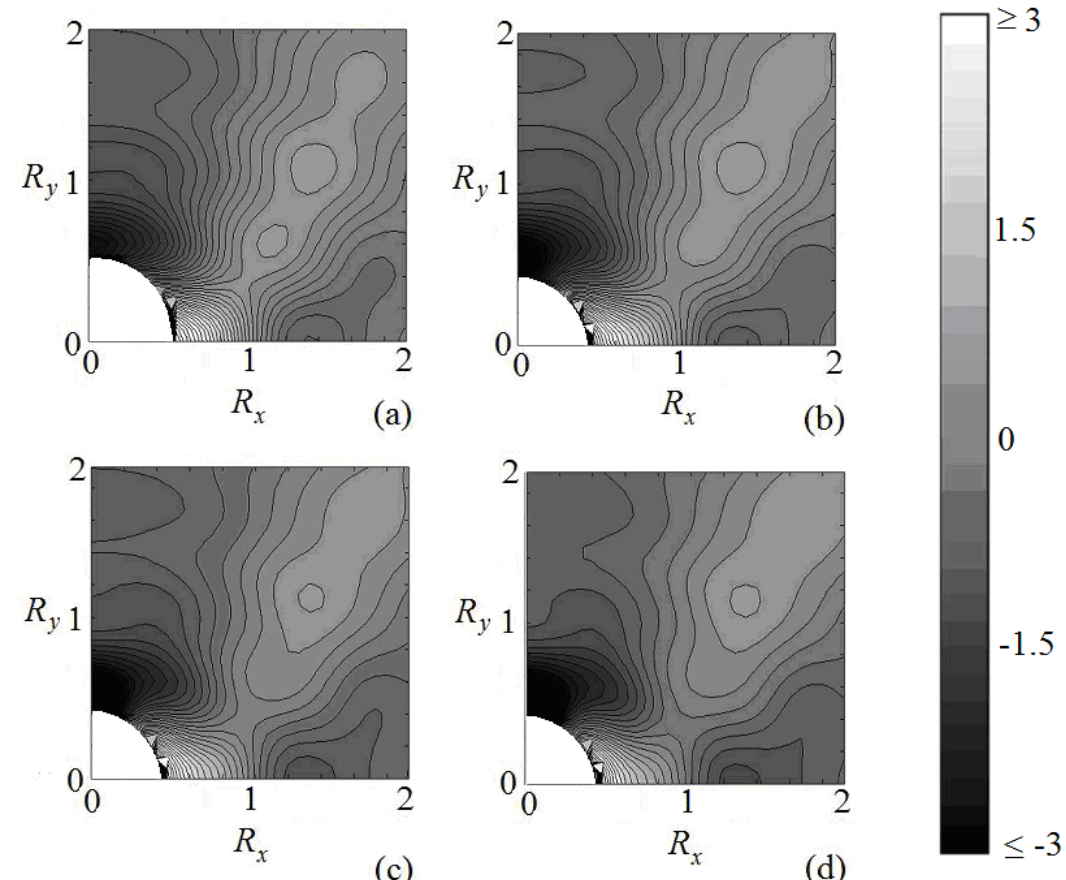

(c)

(d)

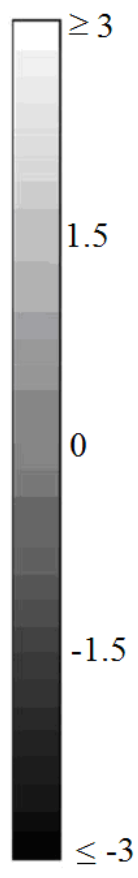

Figure 4. Contour map of the optical binding energy on the $x y$-plane when $\mathbf{E}_{2}$ is parallel to the $y$-axis (See set-up in Fig. 3). For the Lorentzian spectra we use $\omega_{0}=3 \gamma, \omega_{r 0}=10 \gamma, \omega_{1}=6 \gamma$ and (a) $K_{s} / K_{p}=0.2$ (b) $K_{s} / K_{p}=0.4$ (c) $K_{s} / K_{p}=0.6$ (d) $K_{s} / K_{p}=0.8$. The units of energy and distance are $100 K_{p} N^{2}\left|\mu^{r 0}\right|^{4} /\left(c \varepsilon_{o} \hbar^{2}\right)$ and $c /(\gamma)$, respectively. 

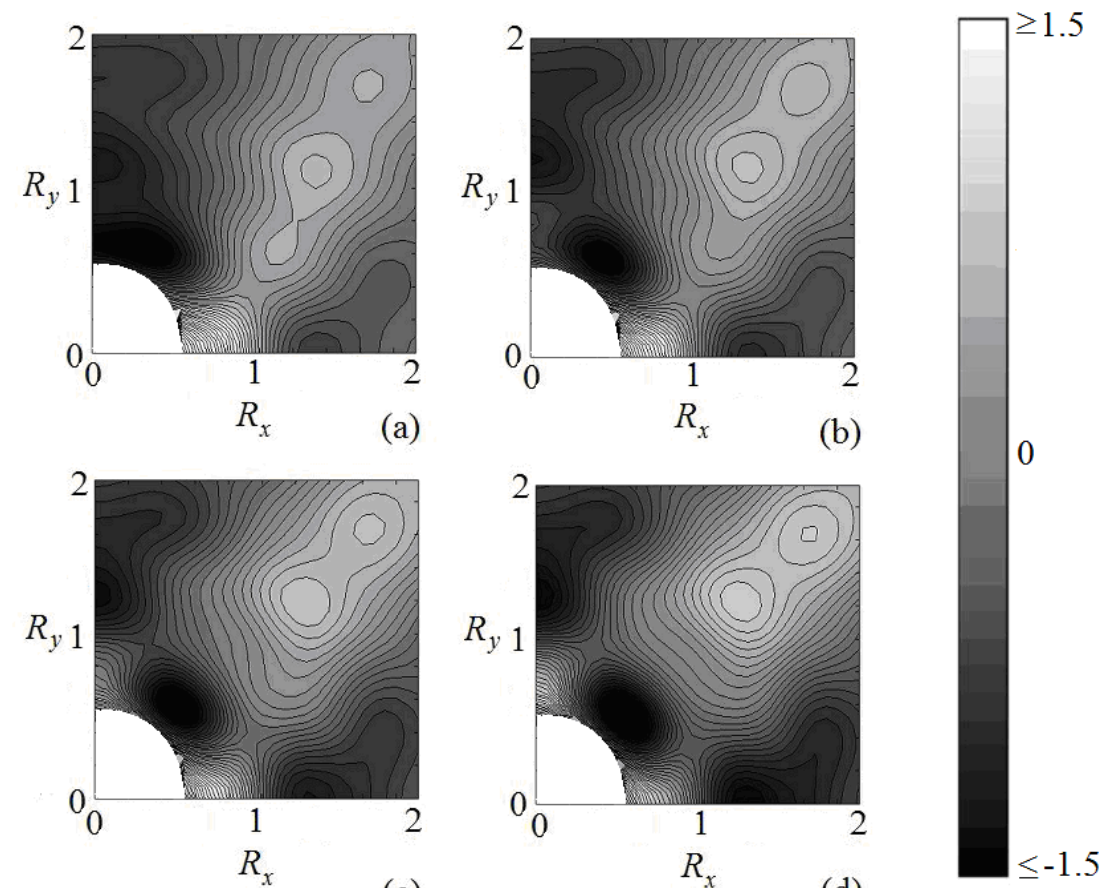

(c)

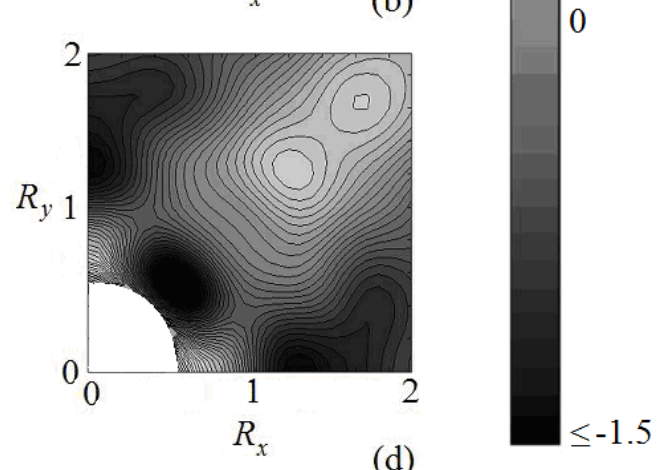

Figure 5. Contour map of the optical binding energy on the $x y$-plane when $\mathbf{E}_{2}$ is parallel to the $z$-axis (See set-up in Fig. 3). For the Lorentzian spectra we use $\omega_{0}=3 \gamma, \omega_{r 0}=10 \gamma, \omega_{1}=6 \gamma$, and (a) $K_{s} / K_{p}=0.25$ (b) $K_{s} / K_{p}=0.5$ (c) $K_{s} / K_{p}=0.75$ (d)

$K_{s} / K_{p}=1$. The units of energy and distance are $100 K_{p} N^{2}\left|\mu^{r 0}\right|^{4} /\left(c \varepsilon_{o} \hbar^{2}\right)$ and $c /(\gamma)$, respectively.

Very different changes to the energy surface are observed when $\mathbf{E}_{2}$ is parallel to the $z$-axis. In this case, with increasing irradiance of the secondary optical field, Fig. 5 (a), (b), (c), and (d), the first energy minimum along the $y$-axis is displaced to an off-axial position. When $K_{s} / K_{p}=1$, Fig. 5 (d), the energy minimum becomes equidistant from the $x$ and $y$-axes, and the surface has an axis of symmetry along $(\hat{x}+\hat{y})$. The position of the second energy minimum along the $y$-axis and the minima along the $x$-axis are not changed significantly, but there is change in form of the potential wells. As a consequence of these changes, if a pair of particles is initially positioned along the $y$-axis, bound by the first minima, the pair is partially rotated upon increasing the irradiance of the secondary field, conserving the initial separation. If a particle is bound by either of the other two above-mentioned energy minima, it does not suffer any change in position. A set-up for the manipulation of particles on a flat surface with optical fields is to be considered in the following section.

\section{OPTICAL BINDING ON A DIELECTRIC SURFACE}

There has been much recent interest in the optical manipulation of particles laying on a surface [32-37]. Non-contact methods are greatly to be favoured for nanoscale particle assembly and surface modifications, obviating contact forces and possible chemical interactions. Here, we consider the possibility of using optical binding for such a purpose. To this end we use a set-up that permits this type of manipulation without the presence of optical pressure. The set-up is shown in Fig. 6; it consists of two beams, mirror images of each other, irradiating a surface on which there are two Rayleigh particles; the surface is oriented with its normal vector along the $z$-axis. The input polarization vectors form an angle $\theta$ with the $x$-axis and the associated Poynting vectors each form an angle $\phi$ with the $y$-axis. Under these conditions, the optical binding energy for a pair of particles can be expressed as follows; 


$$
\begin{aligned}
& \left\langle\Delta E_{\text {avg }}\right\rangle=\operatorname{Re}\left[\frac{\varepsilon}{2 c} \int S(\omega)(\mathbf{e} \cdot \mathbf{V}(\omega, \mathbf{R})) \cdot \mathbf{e} \times\right. \\
& \left.\left(\chi^{(A)}(\omega) \chi^{(B)}(-\omega) e^{i\left(\frac{\omega\left(r_{x}^{(A)}-r_{x}^{(B)}\right) \cos \phi}{c}\right)}+\chi^{(A)}(-\omega) \chi^{(B)}(\omega) e^{-i\left(\frac{\omega\left(r_{x}^{(A)}-r_{x}^{(B)}\right) \cos \phi}{c}\right)}\right]\right) d \omega,
\end{aligned}
$$

where $\mathbf{e}=(\sin \theta \sin \phi, \cos \theta, \sin \theta \cos \phi)$, and the elements of the tensor $\mathbf{V}(\omega, \mathbf{R})$ are defined in equation (2); $S(\omega)$ is the total irradiance that the particles experience; the susceptibilities are again given by equation (5); In the following calculations the system is assumed to be in vacuum. By modifying the two angles, the structures of stable particle arrays on the flat surface are modified.

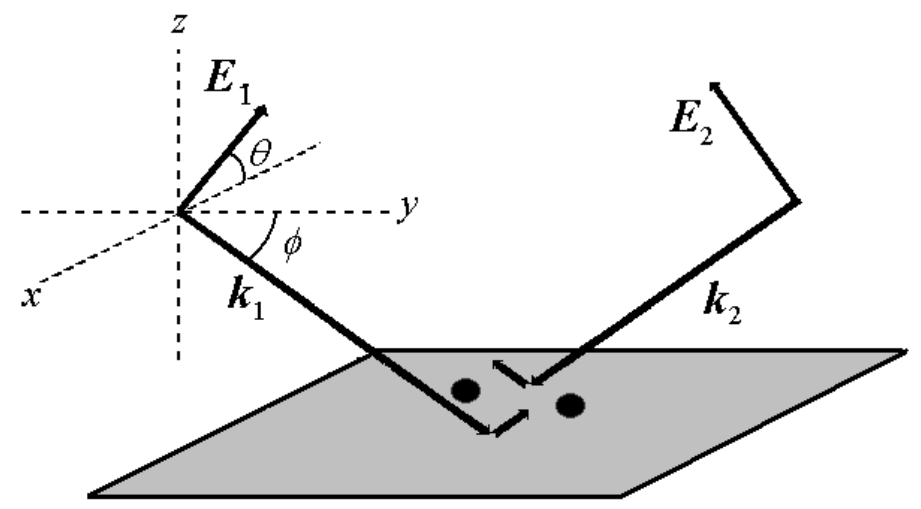

Figure 6. Geometry of two beams, being mirror images of each other, for the manipulations of particles on a surface. The Poynting vectors lay on the $x z$-plane, and the beams are reflected.

Fig. 7 shows the optical binding energy in the near-field zone. In this region, the inter-particle potential is inversely proportional to the cube of the particle separation, and consequently it rapidly diminishes in magnitude with any increase in this distance. The beams here point towards the flat surface, $\phi=90$, and an attractive force in the direction of the polarization is generated. By passively rotating the polarization (i.e. by changing $\theta$ ) a particle pair or chain can be rotated. When the polarizations are perpendicular to the surface, the interaction in the near-field zone becomes repulsive in all directions.

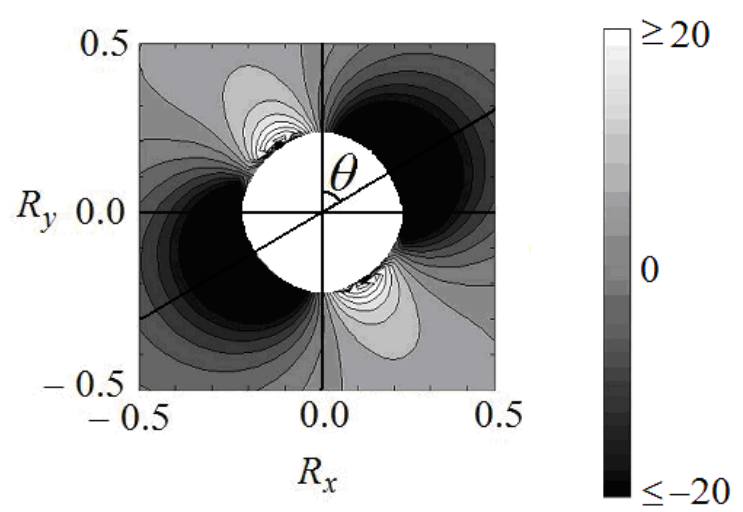

Figure 7. Contour map of the optical binding energy in the near-field zone on the $y z$-plane for $\phi=90$. The energy surface rotates with the value of $\theta$. For the Lorentzian spectrum $\omega_{0}=3 \gamma, \omega_{r 0}=10 \gamma$ and $\omega_{1}=6 \gamma$. The units of energy and distance are

$$
100 K N^{2}\left|\mu^{r 0}\right|^{4} /\left(c \varepsilon_{o} \hbar^{2}\right) \text { and } c /(\gamma) \text {, respectively. }
$$



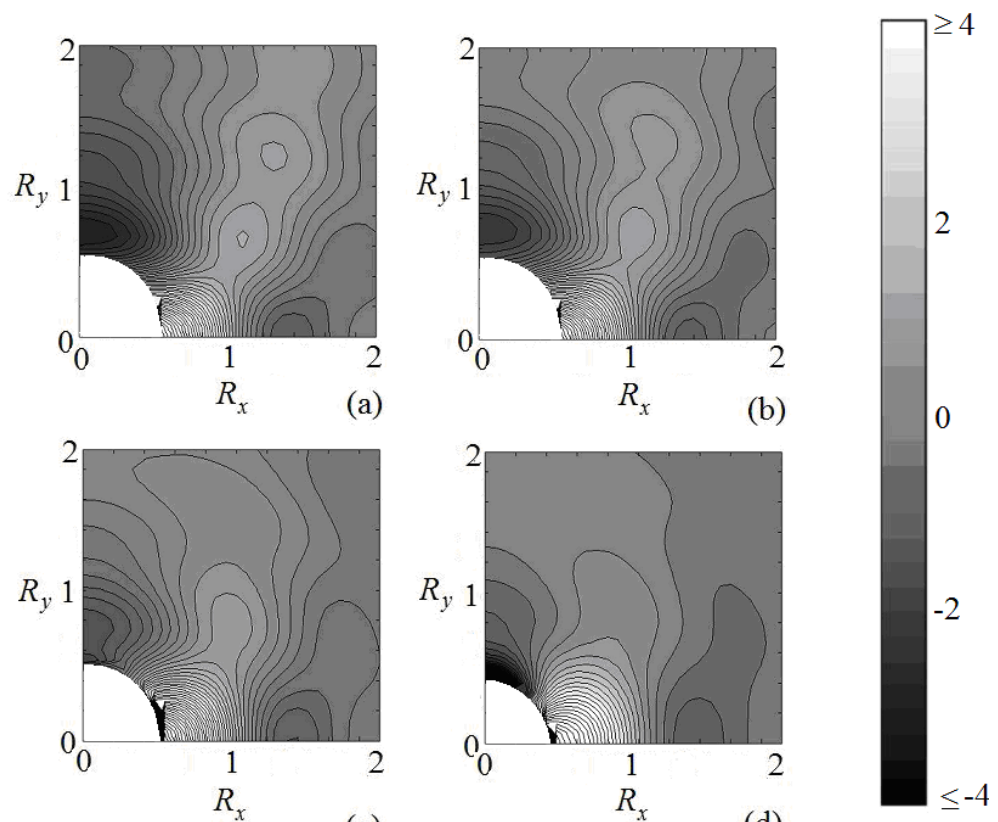

(c)

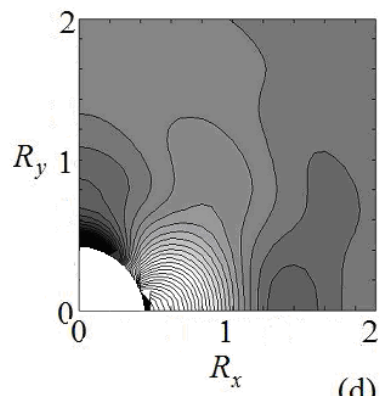

(d)

Figure 8. Contour map of the optical binding energy on the $x y$-plane for $\theta=90$ and (a) $\phi=30$ (b) $\phi=40$ (c) $\phi=50$ (d) $\phi=60$. For the Lorentzian spectrum we use $\omega_{0}=3 \gamma, \omega_{r 0}=10 \gamma$ and $\omega_{1}=6 \gamma$. The units of energy and distance are $100 K N^{2}\left|\mu^{r 0}\right|^{4} /\left(c \varepsilon_{o} \hbar^{2}\right)$ and $c /(\gamma)$, respectively.
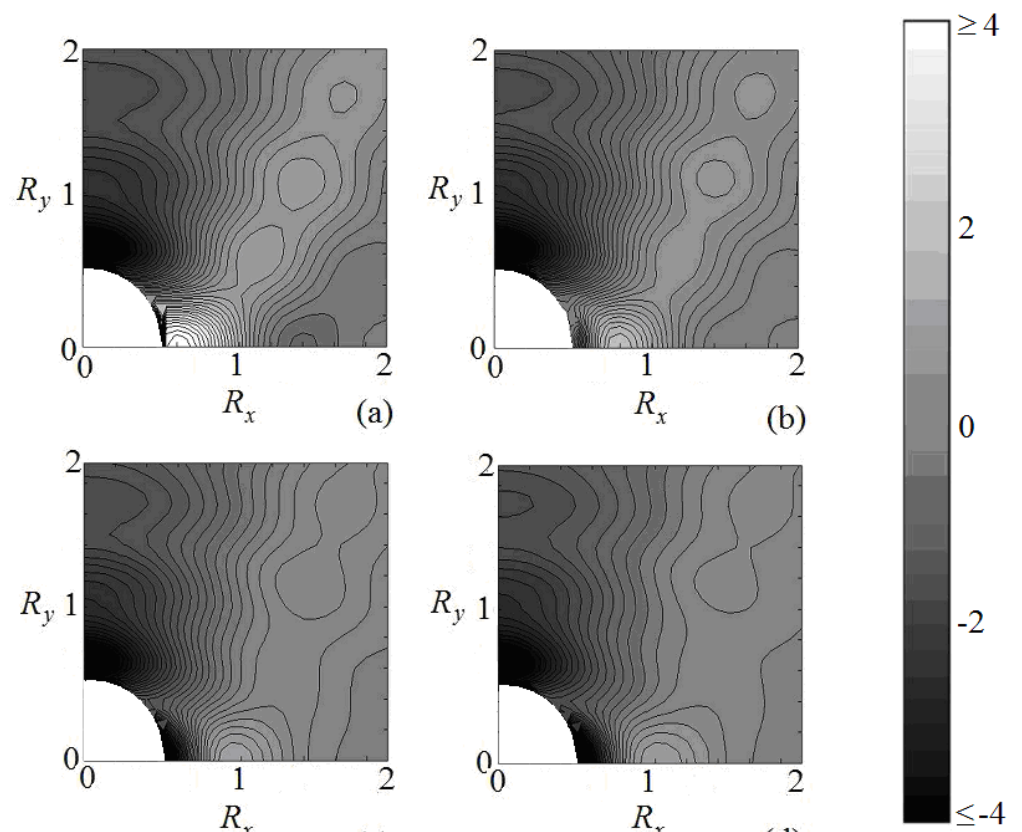

(c)

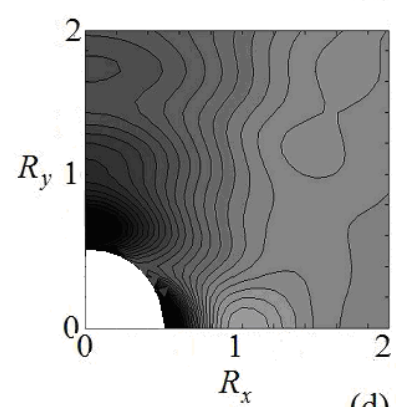

(d)

Figure 9. Contour map of the optical binding energy on the $x y$-plane for $\phi=0$ and (a) $\theta=60$ (b) $\theta=40$ (c) $\theta=20$ (d) $\theta=0$. For the Lorentzian spectrum $\omega_{0}=3 \gamma, \omega_{r 0}=10 \gamma$ and $\omega_{1}=6 \gamma$. The units of energy and distance are $100 K N^{2}\left|\mu^{r 0}\right|^{4} /\left(c \varepsilon_{o} \hbar^{2}\right)$ and $c /(\gamma)$, respectively. 
Fig. 8 illustrates the effect of moving the Poynting vector from the $x$-axis towards the $z$-axis on the particle arrays in the setting illustrated in Fig. 6. In this rotation of the Poynting vector, an effect similar to the one observed in Fig. 4 is exhibited. The potential well of the first stable array along the $y$-axis becomes deeper, and the position of its minimum moves to the near-field zone. Although there are other changes in the form of the concavity along the $x$-axis, the position of the energy minimum does not change significantly.

Fig. 9 shows the effect of rotating the polarization of the beams from the $z$-axis to the $x$-axis while the Poynting vector is along the $y$-axis. During this rotation, the energy maximum on the $x$-axis moves from the near-field zone in Fig. 9 (a) to around $\mathbf{R}=(c /(n \gamma), 0,0)$ in Fig. 9 (d). Also with this polarization rotation, the separation between a particle pair bound by the energy minimum on $\mathbf{R}=(1.5 c /(n \gamma), 0,0)$ is increased. If a particle is initially trapped by the near-field attractive potential along the $x$-axis in Fig. 9 (d), and the polarization is rotated towards the $z$-axis, the particle may eventually escape to the energy minimum along the first $y$-axis - or the one along the $x$-axis - since initially the attractive near-field potential along the $x$-axis becomes completely repulsive when the polarization is parallel to the $z$-axis.

\section{CONCLUSIONS}

A comprehensive analysis has been effected of various techniques for the manipulation of Rayleigh particle arrays using optical binding in broadband light. Three methods have been demonstrated to passively reorder particles arrays in a diversity of forms. One of the methods involves filtering the broadband spectrum of the light, allowing a modification in size of the particle array. A second method uses radiation with transverse Poynting vectors; we have shown that with suitable orientation of the polarization, particle chains may be collapsed or rotated. In a third method, a pair of beams is used to rotate, separate and attract particle pairs laying on a flat surface.

When analysing the effect of the particle size on the optical binding, it has been shown that the potential energy surfaces are continuously modified from the one for Rayleigh particles, to an energy surface where the only significantly stable pair array is two particles, separated by about the diameter of the spheres, disposed along the Poynting vector of the radiation [38]. It is expected that in this continuous transformation of the surface there is a range of particle size in which many of the effects described here may be observed. However, when particles are of around the size of the average wavelength or higher, the modification of the surface under the conditions considered here needs to be studied further. Moreover, if one considers particles on a metal surface, it can be anticipated that the engagement of surface plasmons will exert a considerable influence on the manifestation of optical binding. The effect of particle size and the arrays formed by multi-particle systems are subjects of ongoing investigation.

\section{REFERENCES}

[1] J. Rodríguez, and D. L. Andrews, "Inter-particle interaction induced by broadband radiation,” Opt. Commun. 282, 2267 (2009).

[2] J. Rodríguez, and D. L. Andrews, "Influence of the state of light on the optically induced interparticle interaction,” Phys. Rev. A 79, 022106 (2009).

[3] T. Thirunamachandran, "Intermolecular interactions in the presence of an intense radiation field," Mol. Phys. 40, $393(1980)$.

[4] M. M. Burns, J.-M. Fournier, and J. A. Golovchenko, “Optical binding," Phys. Rev. Lett. 63, 1233 (1989).

[5] P. C. Chaumet and M. Nieto-Vesperinas, "Optical binding of particles with or without the presence of a flat dielectric surface,” Phys. Rev. B 64, 035422 (2001).

[6] S. A. Tatarkova, A. E. Carruthers, and K. Dholakia, "One-Dimensional Optically Bound Arrays of Microscopic Particles," Phys. Rev. Lett. 89, 283901 (2002).

[7] J. Ng, Z. F. Lin, C. T. Chan, and P. Sheng, "Photonic clusters formed by dielectric microspheres: Numerical simulations," Phys. Rev. B 72, 085130 (2005).

[8] M. Guillon, O. Moine, and B. Stout, "Longitudinal Optical Binding of High Optical Contrast Microdroplets in Air," Phys. Rev. Lett. 96, 143902 (2006). 
[9] N. K. Metzger, E. M. Wright, and K. Dholakia, "Theory and simulation of the bistable behaviour of optically bound particles in the Mie size regime," New J. Phys. 8, 139 (2006).

[10] N. K. Metzger, E. M. Wright, W. Sibbett, and K. Dholakia, "Visualization of optical binding of microparticles using a femtosecond fiber optical trap," Opt. Express 14, 3677 (2006).

[11] T. Cizmar, V. Kollarova, Z. Bouchal, and P. Zemánek, "Sub-micron particle organization by self-imaging of nondiffracting beams," New J. Phys. 8, 43 (2006).

[12] V. Karásek, K. Dholakia, and P. Zemánek, "Analysis of optical binding in one dimension," Appl. Phys. B 84, 149 (2006).

[13] F. Chen, G. L. Klimchitskaya, V. M. Mostepanenko, and U. Mohideen, "Demonstration of optically modulated dispersion forces," Opt. Express 15, 4823 (2007).

[14] J. Rodríguez, L. C. Dávila Romero and D. L. Andrews, "Optically induced potential energy landscapes," J. Nanophotonics 1, 019503 (2007).

[15] N. K. Metzger, R. F. Marchington, M. Mazilu, R. L. Smith, K. Dholakia, and E. M. Wright, "Measurement of the Restoring Forces Acting on Two Optically Bound Particles from Normal Mode Correlations," Phys. Rev. Lett. 98, 068102 (2007).

[16] R. G. Crisp and D. L. Andrews, “Optical electrostriction,” Proc. SPIE 6483, 648304 (2007).

[17] S. Ahlawat, R. Dasgupta, and P. K. Gupta, "Optical trapping near a charged surface: three-dimensional optical binding of colloids," Proc. SPIE. 6535, 65350W (2007).

[18] V. Karásek, P. Zemánek, "Analytical description of longitudinal optical binding of two spherical nanoparticles," J. Opt. A 9, S215 (2007).

[19] D. L. Andrews and J. Rodríguez, "Collapse of optical binding under secondary irradiation," Opt. Lett. 33, 1830 (2008).

[20] D. M. Gherardi, A. E. Carruthers, T. Cizmar, E. M. Wright, and K. Dholakia, "A dual beam photonic crystal fiber trap for microscopic particles," Appl. Phys. Lett. 93, 041110 (2008).

[21] J. Rodríguez, L. C. Dávila Romero, and D. L. Andrews, "Optical binding: potential energy landscapes and QED," Proc. SPIE 6905, 69050H (2008).

[22] J. Rodríguez, L. C. Dávila Romero and D. L. Andrews, "Optical binding in nanoparticle assembly: Potential energy landscapes," Phys. Rev. A 78, 043805 (2008).

[23] L. C. Dávila Romero, J. Rodríguez and D. L. Andrews, "Optically induced multi-particle structures: multidimensional energy landscapes," Proc. SPIE 6988, 69880L (2008).

[24] M. Guillon and B. Stout, "Optical trapping and binding in air: Imaging and spectroscopic analysis," Phys. Rev. A 77, 023806 (2008).

[25] M. Kawano, J. T. Blakely, R. Gordon, and D. Sinton, "Theory of dielectric micro-sphere dynamics in a dualbeam optical trap," Opt. Express 16, 9306 (2008).

[26] M. Li and J. Arlt, "Trapping multiple particles in single optical tweezers," Opt. Commun. 281, 135 (2008).

[27] R. F. Marchington, M. Mazilu, S. Kuriakose, V. Garcés-Chávez, P. J. Reece, T. F. Krauss, M. Gu, and K. Dholakia, "Optical deflection and sorting of microparticles in a near-field optical geometry," Opt. Express 16, $3712(2008)$.

[28] V. Karásek, T Cizmár, O. Brzobohatý, P. Zemánek, V. Garcés-Chávez, K. Dholakia, "Long-Range OneDimensional Longitudinal Optical Binding,” Phys. Rev. Lett. 101, 143601 (2008).

[29] V. Karásek, O. Brzobohatý and P. Zemánek, "Longitudinal optical binding of several spherical particles studied by the coupled dipole method," Opt. A: Pure Appl. Opt. 11, 034009 (2009).

[30] R. A. Nome, M. J. Guffey, N. F. Scherer, and S. K. Gray, "Plasmonic Interactions and Optical Forces between Au Bipyramidal Nanoparticle Dimers," J. Phys. Chem. A, 113, 4408 (2009).

[31] R. Loudon, [The Quantum Theory of Light], third ed., Oxford University Press, New York, (2000).

[32] V. Garces-Chavez, K. Dholakia, and G. C. Spalding, "Extended-area optically induced organization of microparticles on a surface," Appl. Phys. Lett. 86, 031106 (2005).

[33] P. J. Reece, V. Garces-Chavez, and K. Dholakia, "Near-field optical micromanipulation with cavity enhanced evanescent waves," Appl. Phys. Lett. 88, 221116 (2006).

[34] L. C. Thomson, G. Whyte, M. Mazilu, and J. Courtial, "Simulated holographic three-dimensional intensity shaping of evanescent-wave fields," J. Opt. Soc. Am. B 25, 849 (2008).

[35] D. Ganic, X. Gan, and M. Gu, "Trapping force and optical lifting under focused evanescent wave illumination," Opt. Express 12, 5533 (2004). 
[36] B. Jia, X. Gan, and M. Gu, "Strong tangential force within a small trapping volume under near-field LaguerreGaussian beam illumination," Opt. Express 16, 15191 (2008).

[37] V. E. Lembessis, M. Babiker and D. L. Andrews, "Surface optical vortices," Phys. Rev. A 79, 011806(R) (2009).

[38] J. Rodríguez, "Quantum electrodynamics analysis of optical binding in counterpropagating beams and effect of particle size" Opt. Lett. 33, 2197 (2008). 